\title{
COPING E GÊNERO EM ADOLESCENTES
}

\author{
Sheila Gonçalves Câmara* \\ Mary Sandra Carlotto
}

\begin{abstract}
RESUMO. Este estudo avalia a associação entre bem-estar psicológico e estratégias de coping nos gêneros masculino e feminino, bem como o perfil diferencial destas entre os gêneros. A amostra foi composta de 389 jovens, estudantes do terceiro ano do ensino médio de escolas de diferentes regiões de Porto Alegre. Os resultados evidenciam que as meninas, ao assumirem uma postura ativa na resolução dos problemas, apresentam maior bem-estar psicológico. Já os meninos apresentam melhor bem-estar na medida em que buscam pertença e procuram amigos íntimos e ajuda profissional. A análise do perfil diferencial entre os gêneros em relação às estratégias de coping nos permite identificar uma função discriminante composta por 9 variáveis, que se distribuem diferencialmente de acordo com a utilização de estratégias referentes a papéis de gênero socialmente construídos..
\end{abstract}

Palavras-chave: enfrentamento, adolescência, gênero.

\section{COPING STRATEGIES AND GENDER IN YOUNG PEOPLE}

\begin{abstract}
The association between psychological well-being and coping strategies in males and females and the differential profile between the genders are evaluated. Sample comprised 389 final year upper junior school young people from different regions in Porto Alegre RS Brazil. Results show young women with a high psychological well-being when they take on an active stance in problem resolution. On the other hand, young men demonstrate a high well-being when they seek a state of belonging, intimate relationships and professional help. Differential profile analysis between genders with regard to coping strategies identifies a discriminating function composed of nine variables. The latter are distributed differentially according to use of strategies involving socially built gender roles.
\end{abstract}

Key words: Coping, young people, gender.

\section{ESTRATEGIAS DE AFRONTAMIENTO (COPING) Y GÉNERO EN ADOLESCENTES}

RESUMEN. Este estudio evalúa la asociación entre bienestar psicológico y estrategias de afrontamiento (coping) en los géneros masculino y femenino, así como el perfil diferencial de éstas entre los géneros. La muestra se compuso de 389 jóvenes, estudiantes de la enseñanza secundaria de la ciudad de Porto Alegre. Los resultados evidencian que las chicas, al asumir una postura activa en la resolución de los problemas, presentan mayor bienestar psicológico. Los chicos, a su vez, presentan mejor bienestar a la medida que buscan pertenencia y buscan amigos íntimos y ayuda profesional. El análisis del perfil diferencial entre los géneros, con relación a las estrategias de afrontamiento (coping) permite identificar una función discriminante, compuesta por 9 variables que se distribuyen diferencialmente, según la utilización de estrategias referentes a roles de género socialmente construidos.

Palabras-clave: afrontamiento (coping), adolescencia, género.

Já existe o consenso de que os fatores psicológicos e sociais podem ocasionar efeitos negativos na saúde orgânica dos indivíduos
(Krantz, 2002). É nesse sentido que as estratégias de coping diante de situações estressantes têm impacto não somente sobre a saúde mental, mas

* Psicóloga, Mestre em Psicologia Social e da Personalidade e Doutora em Psicologia (PUCRS), Professora e Pesquisadora do Laboratório de Ensino e Pesquisa em Psicologia do Curso de Psicologia e do Programa de Pós-Graduação em Saúde Coletiva Universidade Luterana do Brasil - ULBRA/Canoas.

\# Psicóloga, Mestre em Saúde Coletiva (ULBRA-RS), Doutora em Psicologia Social (USC/ES); Professora e Pesquisadora do Laboratório de Ensino e Pesquisa em Psicologia do Curso de Psicologia - Universidade Luterana do Brasil-ULBRA/Canoas. 
também sobre a saúde física e o bem-estar social (Byrne, 2000).

Coping refere-se a um processo oriundo da interação do indivíduo com seu ambiente, na qual ocorre uma série de situações estressantes específicas, provenientes tanto do meio externo quanto do interno, com as quais este indivíduo precisa lidar (Folkman \& Lazarus, 1980). Conforme Blalock e Joiner (2000), consiste em um moderador entre eventos de vida negativos e o impacto de tais eventos no bem-estar físico e psicológico do indivíduo.

Segundo Folkman e Lazarus (1980), para fazer frente às situações estressantes da vida são mobilizados recursos das dimensões cognitiva e comportamental, de acordo com a orientação do indivíduo sobre o problema. Blalock e Joiner (2000) desdobraram estas duas dimensões iniciais, propostas por Folkman e Lazarus (1980), em quatro categorias: aproximação comportamental (realização de uma ação concreta para afrontar a situação estressante ou suas consequiências); aproximação cognitiva (realização de uma análise lógica da situação, reavaliação positiva ou ensaio mental de ações alternativas); evitação comportamental (envolvimento em comportamentos impulsivos, redutores de tensão); e evitação cognitiva (pensamentos ou respostas que objetivam negar ou minimizar a gravidade da situação de crise ou suas conseqüências).

A utilização destas dimensões de enfrentamento de problemas estará associada tanto aos recursos pessoais de coping (habilidades aprendidas no e do ambiente) quanto aos socioecológicos (condições e recursos do ambiente em interação com o indivíduo). A partir destas disposições o evento estressante é submetido a uma avaliação na qual o sujeito busca estratégias que visam equilibrar favoravelmente a relação custobenefício (Antoniazzi, Dell' Aglio \& Bandeira, 1998).

Um dos aspectos a serem considerados em termos de coping é a sua dimensão situacional, ou seja, os esforços que são desenvolvidos pelo indivíduo para enfrentar situações específicas sem perder de vista as condições ou características do contexto (Folkman, Lazarus, Dunkel-Schetter, DeLongis \& Gruen, 1986).

As estratégias de coping parecem ser efetivas na redução das dificuldades ambientais, da mesma forma que contribuem para um melhor ajuste do indivíduo aos problemas que encontra. A maneira como as dificuldades são enfrentadas influencia diretamente os níveis de saúde/bem-estar psicológico; entretanto, na adolescência, muitas estratégias possíveis ainda não foram plenamente desenvolvidas (Williams \& Macgillicuddy-De Lisi, 2000).
$\mathrm{Na}$ medida em que o indivíduo cresce e vai experimentando outras contingências da vida vai aprendendo e testando novas maneiras de lidar com os problemas, uma vez que a etapa da adolescência é um momento de desenvolvimento da identidade, portanto, de um amadurecimento para afrontar as dificuldades (D’Amico, 2000).

Além disso, é preciso considerar a importância de fatores demográficos, pessoais, socioculturais e ambientais, entre outros, que influem no tipo de estratégias de coping a ser mobilizado (Piko, 2001). Nesse processo de experimentação, é provável que, embora involuntariamente, o jovem se defronte com situações para as quais não apresenta repertório consolidado. Estudos recentes revelam a associação entre idade e as tarefas evolutivas que a sociedade apresenta para o adolescente, exigindo novas e diversificadas estratégias de enfrentamento (Scandrolio $\&$ cols, 2002). Nesse sentido, a falta de um repertório adequado pode aumentar a probabilidade de envolvimento em comportamentos de risco, sendo estes percebidos como estratégias para enfrentar situações novas. Da mesma forma que a idade, o gênero se constitui em um fator sociodemográfico crucial para coping e sua relação com a saúde (Piko, 2001).

Quanto ao gênero, não se podem deixar de considerar os padrões ainda vigentes na sociedade brasileira, que atribuem ao gênero masculino a razão, o controle e a liberdade, enquanto à mulher é atribuída uma postura mais passiva e conformista. Esses padrões, mais que regras de comportamento, acabam por criar normas, valores, percepções e representações que constroem e definem a identidade dos indivíduos. Quando os padrões de gênero são assumidos como prototípicos, podem interferir negativamente na saúde dos indivíduos, uma vez que pode constituir-se em uma tarefa bastante árdua o cumprimento de papéis estereotipados do que é ser homem ou mulher (Taquette, Vilhena \& Campos de Paula, 2004).

A questão do gênero é, destarte, bastante ampla e se origina de toda uma rede de relações e influências sociais que englobam determinantes psicossociais e socioestruturais. Assim, não é apenas um modelo de transmissão familiar, mas decorre da transmissão social advinda dos múltiplos sistemas presentes na vida cotidiana (Bussey \& Bandura, 1999).

Não obstante, é preciso considerar que as regras de papel para cada gênero variam em diferentes contextos sociais e momentos da vida. O papel de gênero poderá ser revisto e negociado pelos sujeitos no decorrer de toda a vida, e não apenas na infância. As mudanças socioculturais e tecnológicas atuais, por 
exemplo, passam a exigir um redimensionamento do que pode ser considerado como conduta apropriada de gênero (Taquette \& cols., 2004; Bussey \& Bandura, 1999).

No que diz respeito ao coping, é possível pensar na relação entre os diferentes tipos de resposta e sua relação com os processos de socialização que se pautam por estereótipos de gênero (Frydenberg, 1997). Nesse sentido, de acordo com Piko (2001), tanto meninas como meninos se utilizam bastante de estratégias focalizadas na emoção durante a adolescência inicial. Após esse período, as meninas continuam apresentando esse estilo, enquanto os meninos passam, justamente, a buscar alternativas de distração da emoção. Assim, enquanto o homem passa a usar formas mais diretas, focalizadas no problema ou mesmo de evitação, especialmente não demonstrando seus sentimentos, a mulher assume uma postura mais sentimental perante os problemas e tende a um raciocínio emocional.

Considerando-se a variável gênero, os estudos apontam uma maior tendência por parte dos homens a utilizar estratégias mais arriscadas. Em pesquisa acerca dos preditores dos comportamentos de risco de enfrentamento violento, conduta sexual de risco e consumo de drogas ilegais, encontrou-se que os jovens do sexo masculino apresentavam maior envolvimento nos comportamentos de enfrentamento violento e conduta sexual de risco, invariavelmente relacionados às expectativas de papel para o sexo masculino, como é o caso da coragem e a força, subjacentes aos enfrentamentos violentos e da masculinidade e virilidade relativas às condutas sexuais de risco. Da mesma forma, estes jovens eram os que apresentavam menores índices de bem-estar psicológico, o que se conjugava com a preocupação em manter uma imagem favorável perante seus grupos de referência (Câmara, 2003).

Atualmente, verifica-se um aumento do estresse entre adolescentes (Piko, 2001). Sendo o coping a maneira como o indivíduo faz frente a esse estresse, é fundamental que se possam identificar as estratégias utilizadas pelos adolescentes de ambos os gêneros e avaliar seu impacto sobre a saúde psicossocial dos jovens. A prevenção e a promoção da saúde do adolescente ancoram-se, em grande medida, na compreensão acerca da maneira como ele enfrenta as demandas estressantes, entre elas as expectativas de papel de gênero (Rohlfs, 1999).

Para atender aos objetivos do estudo, realizou-se uma investigação de delineamento analítico preditivocorrelacional, de corte transversal. Verificou-se a relação entre coping e bem-estar psicológico em meninos e meninas e identificou-se o perfil discriminante relativo às estratégias utilizadas por adolescentes de ambos os gêneros. A partir dos resultados buscou-se compreender como as diferenças se apresentam pontualmente para cada gênero. Dessa forma, é possível pensar numa melhor adequação das abordagens utilizadas junto aos jovens de cada gênero, o que também contribui para o desenvolvimento de mecanismos mais adaptativos e saudáveis.

\section{MÉTODO}

\section{Amostra}

A amostragem foi realizada através de procedimento polietápico. Inicialmente, a cidade de Porto Alegre/Brasil foi dividida em 7 regiões, por critérios socioeconômicos e de densidade populacional. Em cada uma das 7 regiões foram sorteadas duas escolas que forneciam ensino médio, uma pública e uma privada. Em cada escola foi sorteada uma turma de $3^{\circ}$ ano do ensino médio. A amostra final foi composta por 389 sujeitos com idade média de 17,3 anos $(D P=1,15)$. Quanto à distribuição por sexo, $41,4 \%$ eram do sexo masculino e $58,6 \%$ do sexo feminino. Dos jovens, 60,7\% estudavam em escolas públicas, sendo que $95 \%$ cursavam o turno diurno.

\section{Instrumentos}

Dados sociodemográficos. Foi organizado questionário especificamente para esta pesquisa, avaliando sexo, idade, tipo de escola (pública ou privada) e turno de estudo (manhã, tarde ou noite). $\mathrm{O}$ questionário era auto-aplicável e os sujeitos marcavam a opção que correspondia a sua condição.

Saúde/Bem-estar-psicológico. General Health Questionnaire - GHQ-12 (Goldberg, 1972). A escala se compõe de 12 itens que são avaliados através de escala tipo Likert de 4 pontos, sendo que quanto menor for o escore do indivíduo, melhor será o seu nível de bem-estar psicológico. A confiança, obtida através do método das duas metades, realizada pelo próprio autor foi de 0,83 para o GHQ-12 (Goldberg, 1972). O GHQ-12 já foi validado em população de jovens de Porto Alegre (Sarriera, Schwarcz \& Câmara, 1996) e obteve um Alfa de 0,80.

Estratégias de coping. Escala de Enfrentamento para Adolescentes - ASC (Frydenberg \& Lewis, 1997). O inventário é composto de 80 itens, sendo o último uma questão aberta na qual o sujeito pode acrescentar outra forma de enfrentamento que utilize e que não esteja relacionada no instrumento. As demais 
79 questões fechadas são respondidas através de escala tipo Likert de 5 pontos, sendo que quanto menores os escores, menor a frequiência de utilização da estratégia. São abarcados 18 fatores que espelham as estratégias de enfrentamento utilizadas pelos adolescentes: apoio social, resolução de problemas, obtenção de êxito, preocupação, busca de relações pessoais íntimas, busca de pertença, esperança e antecipação de saídas positivas, estratégia de falta de coping, redução da tensão, ação social, ignorar o problema, autoculpar-se, reserva, busca de apoio espiritual, fixar-se no positivo, busca de ajuda profissional, busca de diversões relaxantes e distração física. A correlação teste-reteste, para fins de validação da escala foi de $0,32(p<0,001)$ (Frydenbeg \& Lewis, 1997).

A ACS foi traduzida e adaptada para o português, em estudo que verificou sua distribuição fatorial na realidade brasileira (Câmara, Sarriera \& Remor, 2002). Este estudo, realizado com jovens estudantes de Porto Alegre, revelou os seguintes coeficientes de consistência interna para os 18 fatores originais da escala: apoio social $(0,67)$, resolução de problemas $(0,62)$, obtenção de êxito $(0,68)$, preocupação $(0,43)$, busca de relações pessoais íntimas $(0,60)$, busca de pertença $(0,56)$, esperança e antecipação de saídas positivas $(0,59)$, estratégia de falta de coping $(0,60)$, redução da tensão $(0,75)$, ação social $(0,51)$, ignorar o problema $(0,74)$, autoculpar-se $(0,80)$, reserva $(0,68)$, busca de apoio espiritual $(0,56)$, fixar-se no positivo $(0,60)$, busca de ajuda profissional $(0,81)$, busca de diversões relaxantes $(0,28)$ e distração física $(0,80)$.

\section{Procedimentos}

Foram realizados, inicialmente, contatos com as escolas sorteadas a fim de obter autorização para a realização da pesquisa entre seus alunos. A aplicação grupal ocorreu nas salas de aula, com tempo médio de duração de 30 minutos.

$\mathrm{Na}$ análise dos dados, inicialmente foi realizada análise inferencial (correlação de Pearson), para verificar a relação entre coping e bem-estar psicológico em ambos os gêneros. Posteriormente, os dados foram avaliados através de análise multivariada, com a qual se identificou o perfil discriminante de uso de estratégias de coping entre os gêneros. A análise discriminante utiliza uma variável de agrupamento (no caso deste estudo: gênero) e busca a combinação linear das variáveis independentes (preditoras) que maximiza a distância (diferença) entre os grupos.

A pesquisa tem aprovação do Comitê de Ética da instituição de afiliação dos pesquisadores, tendo sido realizados os procedimentos éticos conforme a
Resolução 196 do Conselho Nacional de Saúde (CNS), no que diz respeito à pesquisa com seres humanos (Ministério da Saúde, 1997).

\section{RESULTADOS}

Com vista a avaliar a associação entre coping e bemestar psicológico em ambos os gêneros, realizou-se a análise de correlação de Pearson. Verifica-se, pela tabela 1, que ambos os grupos evidenciam associação, no mesmo sentido, entre bem-estar psicológico e sete das estratégias de enfrentamento estudadas. As estratégias de esforçar-se e ter êxito; fixar-se no positivo e distração física associamse a maiores níveis de bem-estar psicológico em ambos os gêneros; já as estratégias de falta de coping; redução de tensão; autoculpar-se e guardar para si relacionam-se a menores níveis de bem-estar psicológico tanto para meninos como para meninas.

As estratégias que se relacionam a bem-estar psicológico de forma distinta para cada um dos grupos são: resolver problemas; amigos íntimos; buscar pertença; ignorar o problema e buscar ajudar profissional. À medida que as meninas buscam resolver problemas, aumenta seu bem-estar psicológico, ao passo que a estratégia de ignorar o problema o faz diminuir. Para os meninos, buscar amigos íntimos, buscar pertença e buscar ajuda profissional são mecanismos que aumentam seu bem-estar psicológico (Tabela 1).

Tabela 1. Correlação entre Estratégias de Coping e Bem-Estar Psicológico entre os Gêneros. Porto Alegre, $2004(n=389)$.

\begin{tabular}{lcc}
\hline Estratégias de coping & \multicolumn{2}{c}{ Bem-estar psicológico } \\
\cline { 2 - 3 } & Meninos $(n=161)$ & Meninas $(n=228)$ \\
\hline Apoio social & $-0,12$ & 0,00 \\
Resolver problemas & $-0,15$ & $-0,18^{* *}$ \\
Esforçar-se e ter êxito & $-0,18^{*}$ & $-0,26^{* *}$ \\
Preocupar-se & 0,03 & 0,04 \\
Amigos íntimos & $-0,18^{*}$ & $-0,08$ \\
Buscar pertença & $-0,18^{*}$ & 0,04 \\
Criar ilusões & 0,00 & 0,09 \\
Falta de coping & $0,40^{* *}$ & $0,49^{* *}$ \\
Redução da tensão & $0,21^{* *}$ & $0,37^{* *}$ \\
Ação social & $-0,06$ & 0,02 \\
Ignorar o problema & 0,13 & $0,23^{* *}$ \\
Auto culpar-se & $0,19^{*}$ & $0,48^{* *}$ \\
Guardar para si & $0,37^{* *}$ & $0,31^{* *}$ \\
Buscar apoio espiritual & $-0,06$ & 0,02 \\
Fixar-se no positivo & $-0,37^{* *}$ & $-0,37^{* *}$ \\
Busca ajuda profissional & $-0,16^{*}$ & $-0,05$ \\
Buscar diversões relaxantes & $-0,00$ & $-0,13$ \\
Distração física & $-0,19^{*}$ & $-0,19^{* *}$ \\
\hline
\end{tabular}


A fim de considerar a influência das estratégias de coping em adolescentes sobre a variável gênero, utilizou-se a análise discriminante, que aproveita a informação conjunta de todas as variáveis de forma simultânea, diferenciando, assim, os grupos estudados.

A função obtida na análise entre ambos os gêneros, por ser única, explica $100 \%$ da variabilidade entre os dois grupos, constituindo-se no total, com uma correlação canônica moderada, de 0,55 . O autovalor da função foi 0,425 e o $\lambda$ de Wilks de 0,701 . $O$ valor de $\lambda$ é moderadamente alto, o que indica que existe bastante sobreposição entre os grupos. No entanto, o valor transformado de $\lambda\left(\chi^{2}=135,560\right)$ tem associado, com 9 graus de liberdade, um $p \leq 0,000$, de forma que se pode rechaçar a hipótese nula de que os grupos têm médias iguais nas variáveis discriminantes (Pardo-Merino \& Ruiz-Díaz, 2002). A função discriminante classifica corretamente $75 \%$ dos sujeitos em seus respectivos grupos (masculino $=119$ sujeitos corretamente classificados, e feminino $=173$ sujeitos corretamente classificados).

$\mathrm{Na}$ tabela 2 encontram-se as variáveis mais relevantes na capacidade discriminativa desta função.

Tabela 2. Estratégias de Enfrentamento que Entraram na Função Discriminante entre os Gêneros Conforme sua Contribuição Discriminativa na Matriz Estrutural. Porto Alegre, 2004 ( $n=389)$.

\begin{tabular}{lc}
\hline \multicolumn{1}{c}{ Variáveis } & Função 1 \\
\hline ACS Redução da tensão &, 432 \\
ACS Distração física &,- 402 \\
ACS Guardar para si &,- 303 \\
ACS Buscar apoio espiritual &, 284 \\
ACS Falta de afrontamento ou não afrontamento &, 255 \\
ACS Fixar-se no positivo &, 239 \\
ACS Resolver problemas &, 205 \\
ACS Ignorar o problema &,- 194 \\
ACS Ação social &,- 116 \\
\hline
\end{tabular}

Na tabela 2 identifica-se uma função discriminante composta por 9 variáveis que representam fatores da ACS. Nesse sentido, redução da tensão $(0,432)$, busca de apoio espiritual $(0,284)$, falta de afrontamento $(0,255)$, fixar-se no positivo $(0,239)$ e resolver problemas $(0,205)$ são estratégias mais utilizadas por indivíduos do sexo feminino. Entre as mais utilizadas por indivíduos do sexo masculino encontram-se distração física $(-0,402)$, guardar para si $(-0,303)$, ignorar o problema $(-0,194)$ e ação social $(-0,116)$. As estratégias que mais diferenciam ambos os grupos, entre as 9 encontradas, são redução da tensão e distração física.
Para conhecer a situação de cada grupo (gênero) analisado, utilizou-se o valor da média ou centróide de cada setor na função discriminante, que foi de $-0,774$ para os meninos e 0,546 para as meninas, informação que indica a posição dos gêneros em relação à função obtida.

\section{DISCUSSÃO}

Os resultados da correlação entre coping e bemestar psicológico revelam que as estratégias de aproximação ao problema, seja em nível cognitivo seja em nível comportamental, contribuem para um índice mais elevado de bem-estar psicológico tanto para meninas quanto para meninos.

Observa-se que para as meninas a estratégia de resolver o problema, isto é, buscar alternativas de solução, mesmo em nível cognitivo, melhora o bemestar psicológico, ao passo que ignorar o problema constitui-se em um aspecto que diminui o bem-estar. A literatura revela que os mecanismos aproximativos representam formas mais saudáveis e eficazes de lidar com situações de estresse. O aumento nos níveis de atividade indica o emprego de diversas tentativas para solucionar o problema, representando um maior bemestar psicológico (Parsons, Frydenberg \& Poole, 1996; Jensen \& Karoly, 1991; Keefe \& Williams, 1990).

Os resultados encontrados em relação aos meninos vão nessa mesma direção, já que as estratégias que se associam a um maior índice de bem-estar psicológico refletem, basicamente, a atividade de busca, quer pelo apoio de amigos íntimos quer por ajuda profissional para a resolução de problemas. Além disso, para os meninos, a estratégia de busca por pertença (pertencer, integrar-se, fazer parte de um grupo de referência) representa um componente positivo para o bem-estar psicológico.

As diferenças encontradas entre os gêneros na associação entre bem-estar psicológico e coping parecem indicar um perfil mais autodirecionado no caso das meninas e um perfil mais voltado para a busca de apoio externo por parte dos meninos. Esta diferença entre o uso de estilos mais introvertidos ou extrovertidos pode estar relacionada a uma socialização estereotipada em relação aos papéis de gênero (Taquette \& cols., 2004), a qual tem tanta força na constituição da identidade de gênero que chega a influir no bem-estar psicológico.

Ao serem avaliadas as variáveis que mais diferenciavam as estratégias de coping utilizadas por adolescentes em ambos os gêneros, encontraram-se variáveis relativas - basicamente, a evitação comportamental e cognitiva. É o caso das duas variáveis que mais discriminam os sujeitos por gênero, como redução da tensão, que é uma estratégia mais utilizada 
pelas meninas, e distração física, mais utilizada pelos meninos. Ambas têm por finalidade alterar o estado emocional, buscando atividades alternativas que desviem do problema (Chapman \& Mullis, 1999).

Embora os dois grupos utilizem um tipo similar de estratégias, as utilizadas por meninos e meninas diferem qualitativamente. As que discriminam os grupos englobam algumas atribuições bastante específicas, especialmente no período da adolescência, em que as diferenças entre os gêneros se manifestam através de identidades diferenciadas.

A redução da tensão, no caso das meninas, é realizada através de comportamentos como chorar e gritar, encontrar uma forma alternativa de aliviar a tensão e mudar a quantidade do que comem e o tempo de dormir. Os meninos, por sua vez, não vão apresentar esse tipo de recurso e acabam guardando para si seus sentimentos e problemas, ou buscando atividades físicas para alcançar o mesmo resultado de evitação comportamental.

Enquanto as meninas se diferenciam por buscarem apoio espiritual, ou seja, buscar na religião ou em livros dessa natureza uma forma de conforto (Frydenberg, 1991), os meninos diferenciam-se por tentar não pensar no problema, tentar ignorá-lo conscientemente, utilizando uma estratégia de evitação cognitiva para não se defrontar com o estresse que dele se origina. As meninas também apresentam evitação cognitiva quando apresentam a estratégia de falta de afrontamento. Esta evitação se traduz por uma postura derrotista, ou seja, a sensação de incapacidade para resolver o problema e de paralisação diante de uma situação problemática, bem como sensação de mal-estar e sintomas físicos de ansiedade (Chapman \& Mullis, 1999; Frydenberg, 1997). Entretanto, junto com esta estratégia, encontra-se a de fixar-se no positivo, isto é, lembrar-se que outras pessoas têm problemas piores, pensar em coisas boas e ter uma visão mais positiva da vida.

Resultados similares em relação às meninas foram encontrados em estudo de Frydenberg (1991), no qual se verificou que elas utilizavam a estratégia de solução de problemas, ou seja, ir às origens do problema, pensar em soluções alternativas e avaliar possibilidades de atuação sempre com uma postura mais otimista ou esperançosa. Já em relação aos meninos, os resultados diferem, porquanto no estudo com adolescentes australianos a autora encontrou que eles utilizavam menos que as meninas a estratégia de suporte social. No presente estudo os meninos demonstram tentar resolver seus problemas a partir da estratégia de ação social. Esta consiste na busca pelo grupo, isto é, juntarse a outras pessoas para tentar fazer frente aos problemas.

Os achados da presente pesquisa indicam que os jovens se sentem amplamente amparados pelo grupo, o que parece natural neste momento em que ele passa mais tempo com os amigos que com a família (Balaguer, Castillo \& Pastor, 2002), acabando aqueles por tornar-se um grupo de referência até mesmo em termos de modelo de desempenho de papéis (Hurrelman, 1989). Assim, os amigos estabelecem uma espécie de pauta comportamental e uma forte fonte de apoio emocional (Collins \& Repinski, 1994). A proximidade de idade e o compartilhamento de referenciais e experiências similares diminuem as distâncias no diálogo, fazendo com que o jovem se sinta mais confortável para dividir com os amigos aspectos importantes de sua vida (Darling, Hamilton \& Niego, 1994; Darmody, 1991).

O perfil discriminante encontrado aponta para a necessidade de se considerarem os padrões relativos a gênero que estão subjacentes à educação destinada a meninos e meninas como um diferencial na maneira como cada grupo tende a lidar com situações estressantes em seu dia-a-dia. Existem diferenças importantes entre os grupos, e estas requerem atenção, especialmente em termos de promoção da saúde do adolescente e prevenção de comportamentos de risco que podem originar-se de um repertório pobre ou pouco saudável de coping. Embora ambos os grupos utilizem estratégias similares, elas diferem qualitativamente na maneira como se apresentam. No caso dos meninos, é preciso ter atenção para a evitação, que pode levar a uma sobrecarga emocional e, dessa forma, a atitudes de alívio da tensão que incorporem comportamentos de risco.

Em relação às meninas, $o$ apelo às emoções se insere em um contexto sociocultural, em que a mulher pode extravasar seus sentimentos, mas em algum ponto, deve aceitar as situações que se apresentam de uma forma mais passiva ou mesmo mística (Frydenberg, 1991). Esta passividade pode repercutir em outras estratégias, como é o caso da busca por substâncias psicoativas, que tem sido importante entre as meninas (Câmara, 2003).

A preocupação com as alternativas utilizadas por adolescentes para lidar com as situações de estresse devese ao fato de que, nesse período do desenvolvimento, os jovens estão testando suas possibilidades e sua capacidade de lidar com os problemas que surgem. É um momento de fragilidade, especialmente num contexto sociocultural que determina os papéis apropriados para cada gênero, não somente no sentido de definição, mas também no de adequação massiva a normas e padrões comportamentais. Rohlfs (1999) defende a importância da atribuição de papéis de gênero para a saúde, indicando que a análise do impacto do estilo de vida sobre a saúde é um ponto fundamental. 


\section{REFERÊNCIAS}

Antoniazzi, A. S., Dell'Aglio, D. D., \& Bandeira, D. R. (1998). O conceito de coping: uma revisão teórica. Estudos de Psicologia, 3(2), 273-294.

Balaguer, I., Castillo, I., \& Pastor, Y. (2002). Los estilos de vida relacionados con la salud en la adolescencia temprana. Em I. Balaguer (Org.), Estilos de vida en la adolescencia (pp. 5-26). Valencia: Promolibro.

Blalock, J. A., \& Joiner, T. E. (2000). Interaction of cognitive avoidance coping and stress in predicting depression/anxiety. Cognitive Therapy and Research, 24 (1), 47-65.

Bussey, K., \& Bandura, A. (1999). Social cognitive theory of gender development and differentiation. Psychological Review, 106, 676-713.

Byrne, B. (2000). Relationships between anxiety, fear, self-esteem, and coping strategies in adolescence. Adolescence, 35(137), 201-215.

Câmara, S. G., Sarriera, J. C., \& Remor, E. A. (2002). Análise fatorial da escala de afrontamento para adolescentes (ACS) em uma amostra de jovens de Porto Alegre. Aletheia, 16, 15-32.

Câmara, S. G. (2003). Comportamentos de risco na adolescência: Enfrentamento violento, conduta sexual de risco e consumo de drogas ilegais. Tese de doutorado não publicada, Pontifícia Universidade Católica do Rio Grande do Sul, Porto Alegre, Brasil.

Chapman, P. L., \& Mullis, R. L. (1999). Adolescent coping strategies and self-esteem. Child Study Journal, 29(1), 69-77.

Collins, W. A., \& Repinski, D. J. (1994). Relationships during adolescence: Continuity and change in interpersonal perspective. In R. Montemayor, G. R. Adams \& T. P. Gullota (Eds.), Personal relationships during adolescence, Vol 6 (pp. 7-36). London: SAGE Publications.

D'Amico, E. J. (2000). Adolescent risk-taking and prevention: Development of a new risk skills training program. The Sciences and Engineering, 60(9), 55-68.

Darling, N., Hamilton, S. F., \& Niego, S. (1994). Adolescents' relations with adults outside the family. In R. Montemayor, G. R. Adams \& T. P. Gullota (Eds.), Personal relationships during adolescence Vol 6 (pp. 216-235). London: SAGE Publications.

Darmody, J. P. (1991). The adolescent personality, formal reasoning, and values. Adolescence, 26(103), 731-742.

Folkman, S., \& Lazarus, R. S. (1980). An analysis of coping in a middle-aged community sample. Journal of Health and Social Behavior, 21, 219-239.

Folkman, S., Lazarus, R.S., Dunkel-Schetter, C., DeLongis, A., \& Gruen, R.J. (1986). Dynamics of stressful encounter: Cognitive appraisal, coping, and encounter outcomes. Journal of Personality and Social Psychology, 50, 992-1003.

Frydenberg, E. (1991). Adolescent coping: The different ways in which boys and girls cope. Journal of Adolescence, 14(2), 119-134.

Frydenberg, E. (1997). Adolescent coping: Theoretical and research perspectives. New York: Routledege.
Frydenberg, E., \& Lewis, R. (1997). Escalas de afrontamiento para adolescentes: Manual. Madrid: Publicaciones de Psicología Aplicada.

Goldberg, D. P. (1972). The detection of psychiatric illness by questionnaire: A technique for the identification and assesment of non-psychotic psychiatric illness. London: Oxford University.

Hurrelman, K. (1989). Human development and health. Berlín: Springer-Verlag.

Jensen, M. P., \& Karoly, P. (1991). Control beliefs, copinf efforts and adjustment to chronic pain. Journal of Counsoulting and Clinical Psychology, 59, 431-438.

Keefe, F. J., \& Williams, D. A. (1990). A comparison of coping estrategies in chronic pain patients in different age group. Journal of Gerontology, 45, 161-165.

Krantz, D. S. (2002). Effects of psychological and social factors on organic disease: A critical assessment of research on coronary heart disease. Annual Review for Psychology, 53, 321-369.

Ministério da Saúde (1997). Conselho Nacional de Saúde. Diretrizes e normas para pesquisa envolvendo seres humanos. Resolução CNS 196/196. Brasília: Ministério da Saúde.

Pardo-Merino, A. \& Ruiz-Díaz, M. (2002). SPSS 11: Guia para el análisis de datos. Madrid: McGraw-Hill.

Parsons, A., Frydenberg, E. \& Poole, C. (1996). Overachievement and coping strategies in adolescents males. The British Journal of Educational Psychology, 66, 109-114.

Piko, B. (2001). Gender differences and similarities in adolescents' ways of coping. The Psychological Record, 51, 223-235.

Rohlfs, D. (1999). La perspectiva de género el estudio de las diferencias y desigualdades en salud [Resumen]. Anais de la I Jornada de la Red de Médicas y Profesionales de la Salud, Madrid, 2, 12-13.

Sarriera, J. C., Schwarcz, C. \& Câmara, S. G. (1996). Bem-estar psicológico: Análise fatorial da Escala de Goldberg (GHQ-12) numa amostra de jovens. Psicologia, Reflexão e Crítica, 9(2), 293-306.

Scandrolio, B., Martínez, J. M., Marín, M. J., López, J. S., Martín, A., San José, M. C., \& Martín, J. M. (2002). Violencia grupal juvenil: Una revisión critica. Psicothema, 14(supl.), 6-15.

Taquette, S. R., Vilhena, M. M., \& Campos de Paula, M. (2004). Doenças sexualmente transmissíveis e gênero: Um estudo transversal com adolescentes no Rio de Janeiro. Cadernos de Saúde Pública, 20(1), 282-290.

Williams, K., \& Macgillicuddy-De Lisi, A. (2000). Coping strategies in adolescents. Journal of Applied Developmental Psychology, 20, 537-549.

Endereço para correspondência: Sheila Gonçalves Câmara. Rua Edmundo Gardolinski, 70, Bairro Boa Vista, CEP 90480130,Porto Alegre-RS. E-mail: scamara@ via-rs.net 